\title{
Artificial Neurons with Arbitrarily Complex Internal Structures
}

\author{
G.A. Kohring \\ C\&C Research Laboratories, NEC Europe Ltd. \\ Rathausallee 10, D-53757 St. Augustin, Germany
}

\begin{abstract}
Artificial neurons with arbitrarily complex internal structure are introduced. The neurons can be described in terms of a set of internal variables, a set activation functions which describe the time evolution of these variables and a set of characteristic functions which control how the neurons interact with one another. The information capacity of attractor networks composed of these generalized neurons is shown to reach the maximum allowed bound. A simple example taken from the domain of pattern recognition demonstrates the increased computational power of these neurons. Furthermore, a specific class of generalized neurons gives rise to a simple transformation relating attractor networks of generalized neurons to standard three layer feedforward networks. Given this correspondence, we conjecture that the maximum information capacity of a three layer feed-forward network is 2 bits per weight.
\end{abstract}

Keywords: artificial neuron, internal structure, multi-state neuron, attractor network, basins of attraction

(Accepted for publication in Neurocomputing.) 


\section{Introduction}

The typical artificial neuron used in neural network research today has its roots in the McCulloch-Pitts [15] neuron. It has a simple internal structure consisting of a single variable, representing the neuron's state, a set of weights representing the the input connections from other neurons and an activation function, which changes the neuron's state. Typically, the activation function depends upon a sum of the product of the weights with the state variable of the connecting neurons and has a sigmoidal shape, although Gaussian and Mexican Hat functions have also been used. In other words, standard artificial neurons implement a simplified version of the sum-and-fire neuron introduced by Cajal 22] in the last century.

Contrast this for a moment to the situation in biological systems, where the functional relationship between the neuron spiking rate and the membrane membrane potential is not so simple, depending as it does on a host of neuron specific parameters [22]. Furthermore, even the notion of a typical neuron is suspect, since mammalian brains consists of many different neuron types, many of whose functional role in cognitive processing is not well understood.

In spite of these counter examples from biology, the standard neuron has provided a very powerful framework for studying information processing in artificial neural networks. Indeed, given the success of current models such as those of Little-Hopfield [14, 8], Kohonen [12] or Rumelhart, Hinton and Williams 20], it might be questioned whether or not the internal complexity of the neuron plays any significant role in information processing. In other words, is there any pressing reason to go beyond the simple McCulloch-Pitts neuron?

This paper examines this question by considering neurons of arbitrary internal complexity. Previous researchers have attempted to study the affects of increasing neuron complexity by adding biologically relevant parameters, such as a refraction period or time delays, to the neuro-dynamics (see e.g. Clark et al., 1985). The problem with such investigations is that they have so far failed to answer the question of whether such parameters are simply an artifact of the biological nature of the neuron or whether the parameters are really needed for higher-order information processing. To date networks with more realistic neurons look more biologically plausible, but their processing power is not better than simpler networks. An additional problem with such studies, is that as more and more parameters are added to the neurodynamics, software implementations becomes too slow to allow one to work with large, realistically sized networks. Although using silicon neurons [16] can solve the computational problem, they introduce their own set of artifacts 
which may add or detract from their processing power.

The approach taken here differs from earlier work by extending the neuron while keeping the neuro-dynamics simple and tractable. In doing so, we will be able to generalize the notion of the neuron as a processing unit, thereby moving beyond the biological neuron to include a wider variety of information processing units. (One has to keep in mind, that the ultimate goal of the artificial neural network program is not to simply replicate the human brain, but to uncover the general principles of cognitive processing, so as to perform it more efficiently than humans are capable of.) As a byproduct of this approach, we will demonstrate a formal correspondence between attractor networks composed of generalized artificial neurons and the common three layer feed-forward network.

The paper is organized as follows: In the next section, the concept of the generalized artificial neuron is introduced and its usefulness in attractor neural networks is demonstrated, whereby the information capacity of such networks is calculated. Section three presents a simple numerical comparison between networks of generalized artificial neurons and the conventional multi-state Hopfield model. Section four discusses various forms that the generalized artificial neuron can take and the meaning to be attached to them. Section five discusses generalized generalized neurons with interacting variables. The paper ends with a discussion on the merits of the present approach. Proofs and derivations are relegated to the appendix.

\section{Generalized Artificial Neurons (GAN)}

Since its introduction in 1943 by McCulloch and Pitts, the artificial neuron with a single internal variable (hereafter referred to as the McCulloch-Pitts neuron) has been a standard component of artificial neural networks. The neuron's internal variable may take on only two values, as in the original McCulloch and Pitts model, or it may take on a continuum of values. Although, even where analog or continuous neurons are used, it is usually a matter of expediency, e.g., learning algorithms such as back-propagation [20] require continuous variables even if the application only makes use of a two state representation.

Whereas the McCulloch-Pitts neuron presupposes that a single variable is sufficient to describe the internal state of a neuron, we will generalize this notion by allowing neurons with multiple internal variables. In particular, we will describe the internal state of a neuron by $Q$ variables.

Just as biological neurons have no knowledge of the internal states of other neurons, but only exchange electro-chemical signals (Shepherd, 1983), 
a generalized artificial neuron (GAN) should not be allowed knowledge of the internal states of any other GAN. Instead, each GAN has a set of, $C$, characteristic functions, $\mathbf{f} \equiv\left\{f_{i}: R^{Q} \rightarrow R, i=1, \ldots, C\right\}$, which provide mappings of the internal variables onto the reals. It is these characteristic functions which are accessible by other GANs. Even though the characteristic functions may superficially resemble the neuron firing rate, no such interpretation need be imposed upon them.

As in the case of McCulloch-Pitts neurons, the time evolution of the internal variables of a GAN are described by a deterministic dynamics. Here we distinguish between the different dynamics of the $Q$ internal variables by defining $Q$ activation functions, $A_{i}$. These activation functions may depend only upon the values returned by the characteristic functions of the other neurons.

A GAN, $\mathcal{N}(\mathbf{Q}, \mathbf{f}, \mathbf{A})$, is thus described by a a set of internal variables, $\mathbf{Q}$, a set of activation functions, $\mathbf{A}$, and set of characteristic functions, $\mathbf{f}$. Note, for the case of McCulloch-Pitts neuron, there is only a single internal variable governed by single activation function taking on one of two values: 0 or 1 , which also doubles as the characteristic function.

Now, to combine these neurons together into a network, we must define a network topology. The topology is usually described by a set of numbers, $\left\{W_{i j}\right\}(i, j=1, \ldots, N)$, called variously by the names couplings, weights, connections or synapses, which define the edges of a graph having the neurons sitting on the nodes. (In this paper we will use the term "weight" to denote these numbers.) Obviously, many different network topologies are definable, each possessing its own properties, therefore, in order to make some precise statements, let us consider a specific topology, namely that of a fully connected attractor network [14, 8]. Attractor networks form a useful starting point because they are mathematically tractable and there is a wealth of information already known about them.

\subsection{Attractor Networks}

For simplicity, consider the case where each of the $Q$ internal variables is described by a single bit, then the most important quantity of interest is the information capacity per weight, $\mathcal{E}$, defined as:

$$
\mathcal{E} \equiv \frac{\text { Number of bits stored }}{\text { Number of weights }}
$$

For a GAN network the number of weights can not simply be the number of $\left\{W_{i j}\right\}$, otherwise it would be difficult for each internal variable to evolve 
independently. The simplest extension of the standard topology is to allow each internal variable to multiply the weights it uses by an independent factor. Hence, instead of $\left\{W_{i j}\right\}$ we effectively have $\left\{W_{i j}^{a}\right\}$, where, $a=1, \ldots, Q$. A schematic of this type of neuron is given in Figure 1. In an attractor network, the goal is to store $P$ patterns such that the network functions as an auto-associative, or error-correcting memory. The information capacity, $\mathcal{E}$, for these types of networks is then:

$$
\begin{aligned}
\mathcal{E} & =\frac{Q P N}{Q N^{2}} \mathrm{bpw} \\
& =\frac{P}{N} \mathrm{bpw}
\end{aligned}
$$

(bpc $\equiv$ bits per weight) As is well known, there is a fundamental limit on the information capacity for attractor networks, namely $\mathcal{E} \leq 2$ bpw [1, 4, 13, 17]. This implies, $P \leq 2 N$.

Can this limit be reached with a GAN? To answer this question, consider the case where the activation functions are simply Heaviside functions, $H$ :

$$
\begin{aligned}
V_{i}^{a}(t+1) & =H\left(\sum_{j \neq i}^{N} J_{i j}^{a} I_{N}^{j}(t)\right) \\
O_{N}^{i}(t+1) & =F\left(V_{i}^{1}(t+1), \ldots, V_{i}^{Q}(t+1)\right)
\end{aligned}
$$

where $H(x)=0$ if $x<0$ and $H(x)=1$ if $x \geq 0 . s_{i}^{a}(t+1)$ represents the the $a$-th internal variable of the $i$-th neuron. The weight to the internal states of the $i$-th neuron does not violate the principle stated above, because the $i$-th neuron still has no knowledge of the internal states of the other neurons and each neuron is free to adjust its own internal state as it sees fit.

In appendix A we use Gardner's weight space approach [4] to calculate the information capacity for a network defined by Eq. 3, where we now take into account the fact that the total number of weights has increased from $N^{2}$ to $Q N^{2}$. Let $\rho$ denote the probability that $s^{a}=0$ and $1-\rho$ the probability that $s^{a}=1$, then $\mathcal{E}$ for Eq. 3 becomes:

$$
\mathcal{E}=\frac{-\rho \ln _{2} \rho-(1-\rho) \ln _{2}(1-\rho)}{1-\rho+\frac{1}{2}(2 \rho-1) \operatorname{erfc}(x / \sqrt{2})} \text { bpw, }
$$

where $x$ is a solution to the following equation:

$$
(2 \rho-1)\left[\frac{e^{-x^{2} / 2}}{\sqrt{2 \pi}}-\frac{x}{2} \operatorname{erfc}(x / \sqrt{2})\right]=(1-\rho) x
$$


and $\operatorname{erfc}(z)$ is the complimentary error function: $\operatorname{erfc}(z)=(2 / \sqrt{\pi}) \int_{z}^{\infty} d y e^{-y^{2}}$.

When $\rho=1 / 2$, i.e., when $s$ has equal probability of being 0 or 1 , then $x=0$ and the information capacity reaches its maximum bound of $\mathcal{E}=2$ bpw. For highly correlated patterns, e.g., $\rho \rightarrow 1$, the information capacity decreases somewhat, $\mathcal{E} \rightarrow 1 /(2 \ln 2)$ bpw, but, more importantly, it is still independent of $Q$.

What we have shown is that networks of GANs store information as efficiently as networks of McCulloch-Pitts neurons. The difference being, that in the former, each stored pattern contains $N Q$ bits of information instead of $N$. Note: we have neglected the number of bits needed to describe the characteristic functions since they are proportional to $Q N$, which for large $N$ is much smaller than the number of weights, $Q N^{2}$.

\section{A Simple Example}

Before continuing with our theoretical analysis, let us consider a simple, concrete example of a GAN network that illustrates their advantages over conventional neural networks. Again, we consider an attractor network composed of GANs. Each GAN has two internal bit-variables $\mathbf{Q}=\left\{s_{1}, s_{2}\right\}$ whose activation functions are given by Eq. 3 and two characteristic functions, $\mathbf{f}=\{g, h\}$. Let $g \equiv q_{1} \otimes q_{2}$ and $h \equiv q_{1}+2 q_{2}$. In the neurodynamics defined by Eq. 3 we will use the function $g$, reserving the function $h$ for communication outside of the network. (There is no reason why I/O nodes should use the same characteristic functions as compute nodes.)

The weights will be fixed using a generalized Hebbian rule [6, 8], i.e.,

$$
W_{i j}^{a}=\sum_{\mu=1}^{P} s_{i}^{a, \mu} f_{j}^{\mu}
$$

Since this GAN has 4 distinct internal states, we can compare the performance of our GAN network to that of a multi-state Hopfield model [19]. Define the neuron values in the multi-state Hopfield network as $s \in\{-3,-1,1,3\}$ and define thresholds at $\{-2,0,2\}$. (For a detailed discussion regarding the simulation of multi-state Hopfield models see the work of Stiefvater and Müller [23].)

Fig. 2 depicts the basins of attraction for these two different networks, i.e., $d_{0}$ is the initial distance from a given pattern to a randomly chosen starting configuration and $\left\langle d_{f}\right\rangle$ is the average distance to the same pattern when the network has reached a fixed point. For both network types, random sets of patterns were used with each set consisting of $P=0.05 \mathrm{~N}$ patterns. 
The averaging was done over all patterns in a given set and over 100 sets of patterns.

There are two immediate differences between the behavior of the multistate Hopfield network and the present network: 1) the recall behavior is much better for the network of GANs, and 2) using the $X O R$ function as a characteristic function when there are an even number of bit variables, results in a mapping between a given state and its anti-state (i.e., the state in which all bits are reversed), for this reason the basins of attraction have a hat-like shape instead of the sigmoidal shape usually seen in the Hopfield model.

This simple example illustrates the difference between networks of conventional neurons and networks of GANs. Not only is the retrieval quality improved, but, depending upon the characteristic function, there is also a qualitative difference in the shape of the basins of attraction.

\section{Characteristic Functions}

Until now the definition of the characteristic functions, $\mathbf{f}$, has been deliberately left open in order to allow us to consider any set of functions which map the internal variables onto the reals: $\mathbf{f} \equiv\left\{f: R^{Q} \rightarrow R\right\}$. In section 2 no restrictions on the $f$ were given, however, an examination of the derivation in appendix A, reveals that the characteristic functions do need to satisfy some mild conditions before Eq. 1 holds:

$$
\begin{array}{cc}
\text { 1) } & |\langle f\rangle| \ll \sqrt{N}, \\
\text { 2) } \quad\left\langle f^{2}\right\rangle \ll N, \quad \text { and } \\
\text { 3) }\left\langle f^{2}\right\rangle-\langle f\rangle^{2} \neq 0 .
\end{array}
$$

The first two conditions are automatically satisfied if $f$ is a so-called squashing function, i.e, $f: R^{Q} \rightarrow[0,1]$.

\subsection{Linear $f$ and Three Layer Feed-Forward Networks}

One of the simplest forms for $f$ is a simple linear combination of the internal variables. Let the internal variables, $s_{i}^{a}(t)$, be bounded to the unit interval, i.e., $s_{i}^{a} \in[0,1]$, and let $J_{i}^{a}$ denote the coefficients associated with the $i$-th neuron's $a$-th internal variable, then $f$ becomes: 


$$
f_{i}(t)=\sum_{a=1}^{Q} J_{i}^{a} s_{i}^{a}(t) .
$$

Provided, $\left|\sum_{a=1}^{Q} J_{i}^{a}\right| \ll \sqrt{N}$, and provided not all $J_{i}^{a}$ are zero, the three conditions in Eq. 77 will be satisfied. Since the internal variables are bounded to the unit interval, let their respective activation functions be any sigmoidal function, $\mathcal{S}$. Then we can substitute $\mathcal{S}$ into Eq. 8 in order to obtain a time evolution equation solely in terms of the characteristic functions:

$$
f_{i}(t)=\sum_{a=1}^{Q} J_{i}^{a} \mathcal{S}\left(\sum_{j \neq i}^{N} W_{i j}^{a} f_{j}(t-1)\right) .
$$

Formally, this equation is, for a given $i$, equivalent to that of a three layer neural network with $N-1$ linear neurons on the input layer, $Q$ sigmoidal neurons in the hidden layer and one linear neuron on the output layer. From the work of Leshno et al.", we know that three layer networks of this form are sufficient to approximate any continuous function $F: R^{N-1} \rightarrow R$ to any degree of accuracy provided $Q$ is large enough. Leshno et al.'s result applied to Eq. 9 shows that at each time step, a network of $N$ GANs is capable of approximating any continuous function $F: R^{N} \rightarrow R^{N}$ to any degree of accuracy.

In section 2.1 the information capacity of a GAN attractor network was shown to be given by the solution of eqs. 1 and 5. Given the formal correspondence demonstrated above, the information capacity of a conventional three layer neural network must be governed by the same set of equations. Hence, the maximum information capacity in a conventional three layer network is limited to 2 bits per weight.

\subsection{Correlation and Grandmother functions}

A special case of the linear weighted sum discussed above is presented by the correlation function:

$$
f_{i}(t)=\frac{1}{Q} \sum_{a=1}^{Q} t_{i}^{a} s_{i}^{a}(t),
$$

where the $\left\{t_{i}^{a}\right\}$ represent a specific configuration of the internal states of $\mathcal{N}(Q, f)$. With this form for $f$, the GANs can represent symbols using the following interpretation for $f$ : as $f \rightarrow 1$, the symbol is present, and as

\footnotetext{
${ }^{1}$ Leshno et al.'s proof is the most general in a series of such proofs. For earlier, more restrictive results see e.g., [3, 10, 9]
} 
$f \rightarrow 0$ the symbol is not present. Intermediate values represent the symbols partial presence as in fuzzy logic approaches. In this scheme, a symbol is represented locally, but the information about its presence in a particular pattern is distributed. Unlike other representational schemes, by increasing the number of internal states, a symbol can be represented by itself. Consider, for example, a pattern recognition system. If $Q$ is large enough, one could represent the symbol for a tree by using the neuron firing pattern for a tree. In this way, the symbol representing a pattern is the pattern itself.

Another example for $f$ in the same vein as Eq. 10 is given by:

$$
f_{i}(t)=\delta_{\left\{s_{i}^{a}(t)\right\},\left\{t_{i}^{a}\right\}},
$$

where, $\delta_{x, y}$ is the Kronecker delta function: $\delta_{x, y}=1$ iff $x=y$. This equation states that $f$ is one when the value of all internal variables are equal to their values in some predefined configuration. A GAN of this type represents what is sometimes called a grandmother cell.

\subsection{Other Forms of $f$}

Obviously, there are an infinite number of functions one could use for $f$, some of which can take us beyond conventional neurons and networks, to a more general view of computation in neural network like settings. Return for a moment to the example discussed in section 3:

$$
f_{i}(t)=\bigotimes_{a=1}^{Q} s_{i}^{a}(t) .
$$

This simply implements the parity function over all internal variables. Its easy to see that $\langle f\rangle=1 / 2$ and $\left\langle f^{2}\right\rangle-\langle f\rangle^{2}=1 / 4$, hence, this form of $f$ fulfills all the necessary conditions. Using the $X O R$ function as a characteristic function for a GAN trivially solves Minsky and Papert's objection to neural networks [18] at the expense of using a more complicated neuron.

Of course Eq. 12 can be generalized to represent any Boolean function. In fact, each $f_{i}$ could be a different Boolean function, in which case the network would resemble the Kauffman model for genomic systems [11], a model whose chaotic behavior and self-organizational properties have been well studied.

\section{$5 \quad$ Neurons with Interacting Variables}

So far we have considered only the case where the internal variables of the GAN are coupled to the characteristic function of other neurons and not 
to each other, however, in principle, there is no reason why the internal variables should not interact. For simplicity consider once again the case of an attractor network. The easiest method for including the internal variables in the dynamics is to expand Eq. 3 by adding a new set of weights, denoted by, $\left\{L_{i}^{a b}\right\}$, which couple the internal variables to each other:

$$
s_{i}^{a}(t+1)=H\left(\sum_{j \neq i}^{N} W_{i j}^{a} f_{j}+\sum_{b \neq a}^{Q} L_{i}^{a b} s_{i}^{b}\right) .
$$

Using the same technique we use in section 2.1, we can determine the new information capacity for attractor networks (see appendix A):

$$
\mathcal{E}=\mathcal{E}_{0} \frac{\left[1+\lambda \sqrt{\frac{\rho(1-\rho)}{\left\langle\phi^{2}\right\rangle-\langle\phi\rangle^{2}}}\right]^{2}}{(1+\lambda)\left(1+\lambda \frac{\rho(1-\rho)}{\left\langle\phi^{2}\right\rangle-\langle\phi\rangle^{2}}\right)},
$$

where, $\mathcal{E}_{0}$ is given by Eq. $1, \lambda \equiv Q / N$ and $\langle\phi\rangle$ is the average value of the characteristic function at the fixed points. From this equation we see that if the fluctuations in the characteristic functions are equal to the fluctuations in the internal variables, then $\mathcal{E}=\mathcal{E}_{0}$, otherwise, $\mathcal{E}$ is always less than $\mathcal{E}_{0}$.

\section{Summary and Discussion}

In summary, we have introduced the concept of the generalized artificial neuron $(\mathrm{GAN}), \mathcal{N}(\mathbf{Q}, \mathbf{f}, \mathbf{A})$, where $\mathbf{Q}$ is a set of internal variables, $\mathbf{f}$ is a set characteristic functions acting on those variables and $\mathbf{A}$ is a set of activation functions describing the dynamical evolution of those same variables. We then showed that the information capacity of attractor networks composed of such neurons reaches the maximum allowed value of 2 bits per weight. If we use a linear characteristic function à la Eq. 8, then we find a relationship between three layer feed forward networks and attractor networks of GANs. This relationship tells us that attractor networks of GANs can evaluate an arbitrary function of the form $F: R^{N} \rightarrow R^{N}$ at each time step. Hence, their computational power is significantly greater than that of attractor networks with two state neurons.

As an example of the increased computation power of the GAN, we presented a simple attractor network composed of four state neurons. The present network significantly out performed a comparable multi-state Hopfield model. Not only were the quantitative retrieval properties better, but the qualitative features of the basins of attraction were also fundamentally 
different. It is this promise of obtaining qualitative improvements over standard models that most sets the GAN approach apart from previous work.

In section 2.1, the upper limit on the information capacity of an attractor network composed of GANs was shown to be 2 bits per weight, while, in section 4.1 we demonstrated a formal correspondence between these networks and conventional three layer feed-forward networks. Evidently, the information capacity results apply to the more conventional feed-forward network as well.

The network model presented here bears some resemblance to models involving hidden (or latent) variables (see e.g., [7]), however, there is one important difference: namely, the hidden variables in other models are only hidden in the sense that they are isolated from the network's inputs and outputs; but they are not isolated from each other, they are allowed full participation in the dynamics, including direct interactions with one another. In our model, the internal neural variables interact only indirectly via the neurons' characteristic functions.

Very recently, Gelenbe and Fourneau [5] proposed a related approach they call the "Multiple Class Random Neural Network Model". Their model also includes neurons with multiple internal variables, however, they do not distinguish between activation and characteristic functions, furthermore, they restrict the form of the activation function to be a stochastic variation of the usual sum-and-fire rule, hence, their model is not as general as the one presented here.

In conclusion, the approach advocated here can be used to exceed the limitations imposed by the McCulloch-Pitts neuron. By increasing the internal complexity we have been able to increase the computational power of the neuron, while at the same time avoiding any unnecessary increase in the complexity of the neuro dynamics, hence, there should be no intrinsic limitations to implementing our generalized artificial neurons. 


\section{References}

[1] Cover, T. M. Capacity problems for linear machines. In Pattern Recognition (New York, 1968), L. Kanal, Ed., Thompson Book Company, pp. 929-965.

[2] Edwards, S. F., And Anderson, P. W. Theory of spin glasses. Journal of Physics F 5 (1975), 965-974.

[3] Funahashi, K. On the approximate realization of continuous mappings by neural networks. Neural Networks 2 (1989), 183-192.

[4] Gardner, E. The space of interactions in neural network models. Journal of Physics A 21 (1988), 275-270.

[5] Gelenbe, E., And Fourneau, J.-M. Random neural networks with mutliple classes of signals. Neural Computation 11 (1999), 953-963.

[6] HeBB, D. O. The Organization of Behavior. John Wiley \& Sons, New York, 1949.

[7] Hinton, G. E., And Sejnowski, T. J. Learning and Relearning in Boltzmann Machines. In Rumelhart et al. [21], 1986, pp. 282-317.

[8] Hopfield, J. J. Neural networks and physical systems with emergent collective computational abilities. Proceedings of the National Academy of Science, USA 79 (1982), 2554-2558.

[9] HorniK, K. Approximation capabilities of multilayer feed-forward networks. Neural Networks 4 (1991), 251-257.

[10] Hornik, K., Stinchcombe, M., And White, H. Multilayer feedforward networks are universal approximators. Neural Networks 2 (1989), 359-366.

[11] Kauffman, S. A. Origins of Order: Self-Organization and Selection in Evolution. Oxford University Press, Oxford, 1992.

[12] Kohonen, T. Self-Organization and Associative Memory. SpringerVerlag, Berlin, 1983.

[13] Kohring, G. A. Neural networks with many-neuron interactions. Journal de Physique 51 (1990), 145-155.

[14] Little, W. A. The existence of persistent states in the brain. Mathematical Bioscience 19 (1974), 101-119. 
[15] McCulloch, W. S., And Pitts, W. A logical calculus of the idea immanent in nervous activity. Bulletin of Mathematical Biophysics 5 (1943), 115-133.

[16] Mead, C. Analog VLSI and Neural Systems. Addison-Wesley, Reading, 1989.

[17] Mertens, S., Köhler, H. M., And Bos, S. Learning grey-toned patterns in neural networks. Journal of Physics A 24 (1991), 49414952.

[18] Minsky, M., And Papert, S. Perceptrons: An Introduction to Computational Geometry. MIT Press, Cambridge, 1969.

[19] Rieger, H. A. Storing an extensive number of gray-toned patterns in a neural network using multi-state neurons. Journal of Physics A 23 (1990), L1273-L1280.

[20] Rumelhart, D. E., Hinton, G. E., and Williams, R. J. Learning representations by error propagation. In Rumelhart et al. [21], 1986, pp. 318-362.

[21] Rumelhart, D. E., McClelland, J. L., and the PDP ReSearch Group, Eds. Parallel Distributed Processing. MIT Press, Cambridge, MA, 1986.

[22] Shepherd, G. M. Neurobiology. Oxford University Press, Oxford, 1983.

[23] Stiefvater, T., And Müller, K.-R. A finite-size scaling investigation for $q$-state hopfield models: Storage capacity and basins of attractions. Journal of Physics A 25 (1992), 5919-5929. 
Figures 


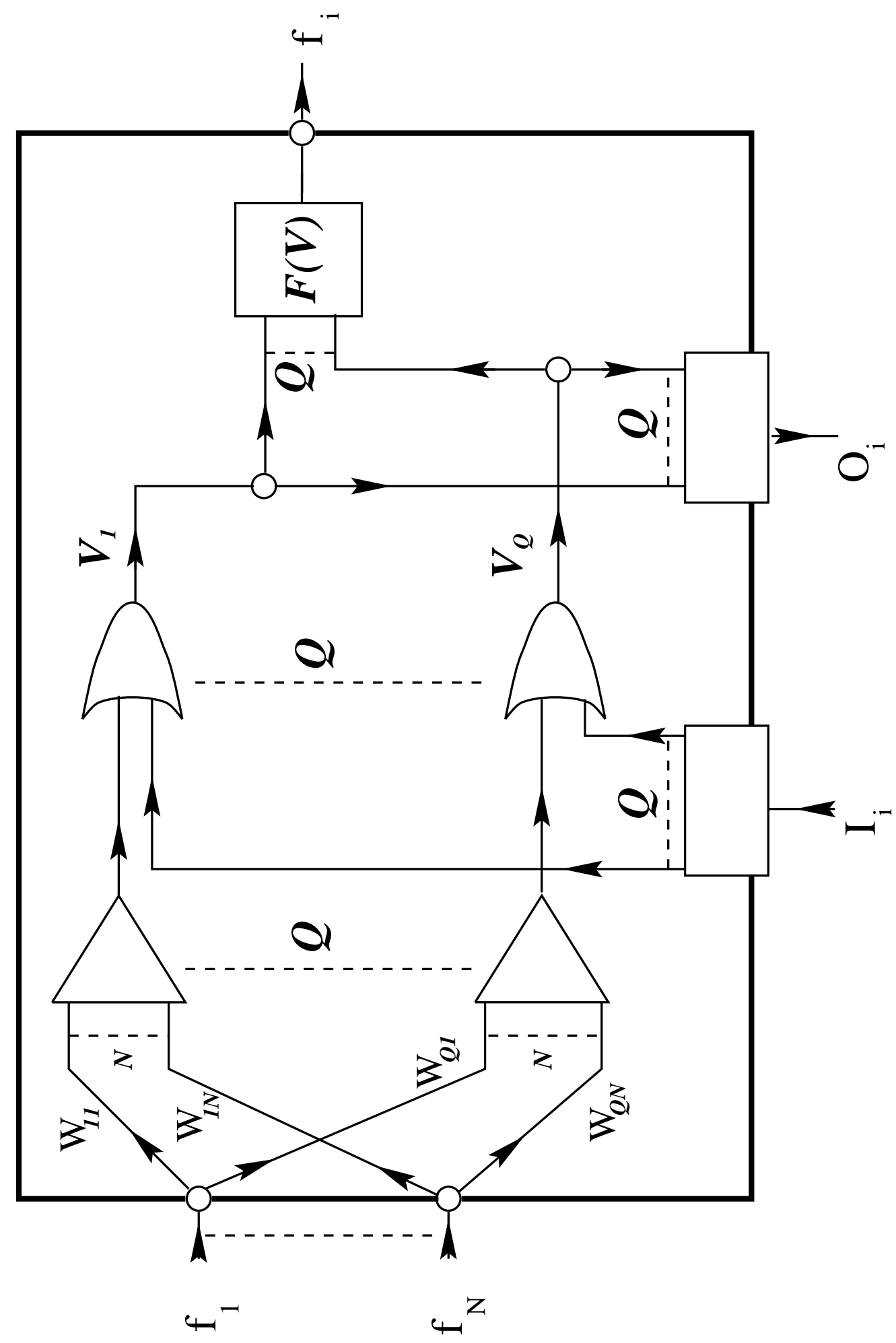

Figure 1: A schematic of a generalized artificial neuron. $f_{i}$ denotes the value of the $i$-th neuron's characteristic function, these are the values communicated to other neurons in the networlk $I_{i}$ and $O_{i}$ denote input and output values used for connections external to the network. 


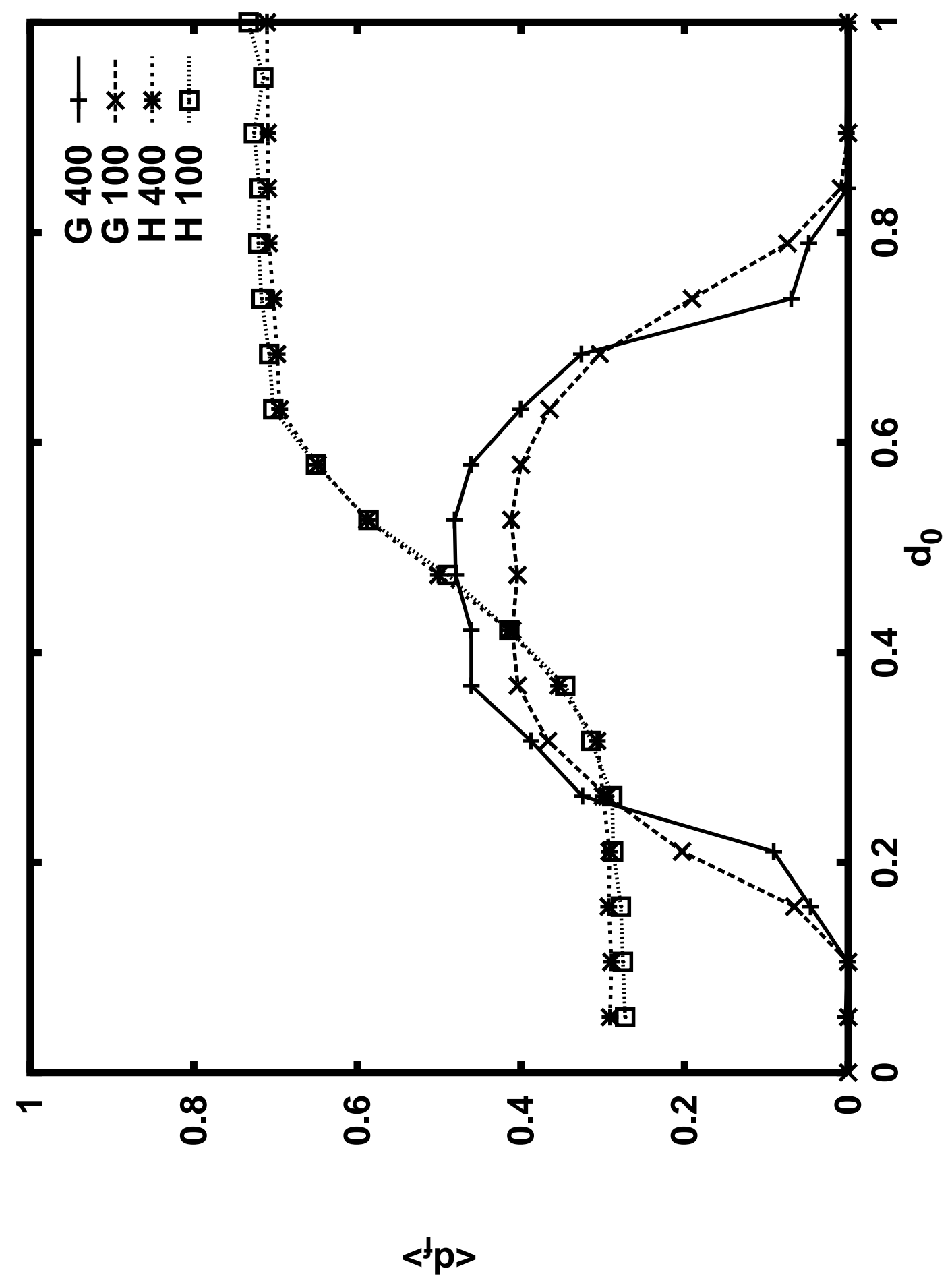

Figure 2: Basins of attraction for a GAN network (lower curves) and for a multi-state Hopfield model (upper curve). In both cases the number of stored patterns is $P=0.05 N$. In edoh case two different system sizes are shown, one with $N=100$ neurons and one with $N=400$ neurons. 


\section{A Derivation of the Information Capacity}

For simplicity consider a homogeneous network of $N$ GANs, where the $Q$ internal variables of each neuron are simply bit-variables. In addition, we will consider the general case of interacting bits. Given $P$ patterns, with $\phi_{i}^{\mu}$ representing the characteristic functions and $\sigma_{i}^{a \mu}$ the internal bit-variables, then by equation eqs. 3 and 13, we see that these patterns will be fixed points if:

$$
\left(2 \sigma_{i}^{a \mu}-1\right)\left(\sum_{j \neq i}^{N} W_{i j}^{a} \phi_{i}^{\mu}+\sum_{b=1}^{Q} L_{i}^{a b} \sigma_{i}^{b \mu}\right)>0 .
$$

In fact, the more positive the left hand side is, the more stable the fixed points. Using this equation we can write the total volume of weight space available to the network for storing $P$ patterns as:

$$
V=\prod_{i, a} V_{i}^{a}
$$

where,

$$
\begin{gathered}
V_{i}^{a}=\frac{1}{Z_{i}^{a}} \int \prod_{j} d W_{i j}^{a} \prod_{b} d L_{i}^{a b} \delta\left(\sum_{j \neq i}^{N}\left(W_{i j}^{a}\right)^{2}-N\right) \delta\left(\sum_{b \neq a}^{Q}\left(L_{i}^{a b}\right)^{2}-Q\right) \times \\
\prod_{\mu} H\left[\left(2 \sigma_{i}^{a \mu}-1\right)\left(\frac{1}{\sqrt{N}} \sum_{j \neq i}^{N} W_{i j}^{a} \phi_{j}^{\mu}+\frac{1}{\sqrt{N}} \sum_{b \neq a}^{Q} L_{i}^{a b} \sigma_{i}^{b \mu}-\theta_{i}^{a}\right)-\kappa\right],
\end{gathered}
$$

and

$$
Z_{i}^{a}=\int \prod_{j} d W_{i j}^{a} \prod_{b} d L_{i}^{a b} \delta\left(\sum_{j \neq i}^{N}\left(W_{i j}^{a}\right)^{2}-N\right) \delta\left(\sum_{b \neq a}^{Q}\left(L_{i}^{a b}\right)^{2}-Q\right) .
$$

where $\kappa$ is a constant whose purpose is the make the left hand side of Eq. 15 as large as possible. (Note, although we have introduce a threshold parameter, $\theta_{i}^{a}$, we will show that thresholds do not affect the results.)

The basic idea behind the weight space approach is that the subvolume, $V_{i}^{a}$, will vanish for all values of $P$ greater than some critical value, $P_{c}$. In order to find the average value of $P_{c}$, we need to average Eq. 17 over all configurations of $\sigma_{i}^{a \mu}$. Unfortunately, the $\sigma_{i}^{a \mu}$ represent a quenched average, which means that we have to average the intensive quantities derivable from $V$ instead of averaging over $V$ directly. The simplest intensive such quantity is: 


$$
\begin{aligned}
\mathcal{F} & =\lim _{N \rightarrow \infty}\left\langle\ln V_{i}^{a}\right\rangle_{\sigma_{i}^{a \mu}}, \\
& =\lim _{\substack{N \rightarrow \infty \\
n \rightarrow 0}} \frac{\left\langle\left(V_{i}^{a}\right)^{n}\right\rangle_{\sigma_{i}^{a \mu}}-1}{n} .
\end{aligned}
$$

The technique for performing the averages in the limit $n \rightarrow 0$ is known as the replica method [2].

By introducing integral representations for the Heaviside functions $\left(H(z-\kappa)=\int_{\kappa}^{\infty} d x \int_{-\infty}^{\infty} d y \exp (i y x) \quad\right)$ we can perform the averages over the $\sigma_{i}^{a \mu}$ :

$$
\begin{aligned}
&\left\langle V_{i}^{a}\right\rangle_{\sigma_{i}^{a \mu}}= \sum_{\sigma_{j}^{b \mu}} \frac{1}{Z_{i}^{a}} \int \prod_{j A} d W_{i j}^{a A} \int_{\kappa}^{\infty} \prod_{\mu A} d x_{\mu}^{A} \int_{-\infty}^{\infty} \prod_{\mu A} d y_{\mu}^{A} \times \\
& \exp \left\{i \sum _ { \substack { A = 1 \\
\mu = 1 } } ^ { n , P } y _ { \mu } ^ { A } \left[x_{\mu}^{A}-\left(2 \sigma_{i}^{a \mu}-1\right)\left(\frac{1}{\sqrt{N}} \sum_{j \neq i}^{N} W_{i j}^{a A} \phi_{j}^{\mu}+\right.\right.\right. \\
&\left.\left.\left.\frac{1}{\sqrt{N}} \sum_{b \neq a}^{Q} L_{i}^{a b A} \sigma_{i}^{b \mu}-\theta_{i}^{a}\right)\right]\right\} \times \\
& \\
& \prod_{A=1}^{n} \delta\left(\sum_{j \neq i}^{N}\left(W_{i j}^{a A}\right)^{2}-N\right) \delta\left(\sum_{b \neq a}^{Q}\left(L_{i}^{a b}\right)^{2}-Q\right) .
\end{aligned}
$$

First sum over the $\sigma_{j}^{b \mu}$ where $j \neq i$ :

$$
\begin{aligned}
& \sum_{\sigma_{j}^{b \mu}} \exp \left\{-i \sum_{\substack{A=1 \\
\mu=1}}^{n, P} y_{\mu}^{A}\left(2 \sigma_{i}^{a \mu}-1\right)\left(\frac{1}{\sqrt{N}} \sum_{j \neq i}^{N} W_{i j}^{a A} \phi_{j}^{\mu}\right)\right\}= \\
& \prod_{j, \mu} \sum_{\sigma_{i}^{a \mu}} \exp \left\{-i \frac{\left(2 \sigma_{i}^{a \mu}-1\right)}{\sqrt{N}} \sum_{A} y_{\mu}^{A} W_{i j}^{a A} \phi_{j}^{\mu}\right\} \approx \\
& \prod_{j, \mu}\left[1-i \frac{\left(2 \sigma_{i}^{a \mu}-1\right)\langle\phi\rangle}{\sqrt{N}} \sum_{A} y_{\mu}^{A} W_{i j}^{a A}-\frac{\left\langle\phi^{2}\right\rangle}{2 N} \sum_{A B} y_{\mu}^{A} y_{\mu}^{B} W_{i j}^{a A} W_{i j}^{a B}\right] \approx \\
& \exp \left\{-i \frac{\left(2 \sigma_{i}^{a \mu}-1\right)\langle\phi\rangle}{\sqrt{N}} \sum_{A \mu} y_{\mu}^{A} \sum_{j} W_{i j}^{a A}-\frac{\left\langle\phi^{2}\right\rangle-\langle\phi\rangle^{2}}{2 N} \sum_{A B} \sum_{\mu} y_{\mu}^{A} y_{\mu}^{B} \sum_{j} W_{i j}^{a A} W_{i j}^{a B}\right\},
\end{aligned}
$$

now sum over the $\sigma_{j}^{b \mu}$ where $j=i$ but $b \neq a$ : 


$$
\begin{aligned}
& \sum_{\sigma_{j}^{b \mu}} \exp \left\{-i \sum_{\substack{A=1 \\
\mu=1}}^{n, P} y_{\mu}^{A}\left(2 \sigma_{i}^{a \mu}-1\right)\left(\frac{1}{\sqrt{N}} \sum_{b \neq a}^{Q} L_{i}^{a b A} \sigma_{i}^{b \mu}\right)\right\} \approx \\
& \exp \left\{-i \frac{\left(2 \sigma_{i}^{a \mu}-1\right)(1-\rho)}{\sqrt{N}} \sum_{A \mu} y_{\mu}^{A} \sum_{b} L_{i}^{a b A}-\frac{\rho(1-\rho)}{2 N} \sum_{A B} \sum_{\mu} y_{\mu}^{A} y_{\mu}^{B} \sum_{b} L_{i}^{a b A} L_{i}^{a b B}\right\},
\end{aligned}
$$

where we have use $\rho$ as the probability that $\sigma=0,\langle\phi\rangle \equiv \sum_{\sigma} \phi(\sigma)$ and $\left\langle\phi^{2}\right\rangle \equiv \sum_{\sigma} \phi(\sigma) \phi(\sigma)$. If we insert Eq. 21 into 20 and define the following quantities: $q^{A B}=(1 / N) \sum_{j} W_{i j}^{a A} W_{i j}^{a B}$ and $r^{A B}=(1 / Q) \sum_{b} L_{i}^{a b A} L_{i}^{a b B}$ for all $A<B$ and $M_{i}^{a A}=(1 / \sqrt{N}) \sum_{j} W_{i j}^{a A}$ and $T_{i}^{a A}=(1 / \sqrt{Q}) \sum_{b} L_{i}^{a b A}$ for all $A$, then Eq. 20 can be rewritten as:

$$
\left\langle V_{i}^{a}\right\rangle_{\sigma_{i}^{a \mu}} \propto \int \prod_{A} d z^{A} d M^{A} d E^{A} d U^{A} d T^{A} d C^{A} \prod_{A<B} q^{A B} F^{A B} r^{A B} H^{A B} e^{N G},
$$

where,

$$
\begin{aligned}
G \equiv & \alpha G_{1}(q, M, T)+G_{2}(F, z, E)+\lambda G_{2}(U, H, C)+i \sum_{A<B} F^{A B} q^{A B}+ \\
& i \lambda \sum_{A<B} H^{A B} r^{A B}+\frac{i}{2} \sum_{A} z^{A}+\frac{i \lambda}{2} \sum_{A} U^{A}+O(1 / \sqrt{N}) .
\end{aligned}
$$

$\alpha \equiv P / N$ and we have introduced another parameter: $\lambda \equiv Q / N$. The functions $G_{1}$ and $G_{2}$ are defined as:

$$
\begin{aligned}
G_{1} \equiv \frac{1}{P} \ln \left\langle\int_{\kappa}^{\infty}\right. & \prod_{\mu A} d x_{\mu}^{A} \int_{-\infty}^{\infty} \prod_{\mu A} d y_{\mu}^{A} \exp \left\{i \sum_{A \mu} y_{\mu}^{A}+\right. \\
& i \sum_{A \mu} y_{\mu}^{A}\left(2 \sigma_{i}^{a \mu}-1\right)\left(\theta^{a}-\langle\phi\rangle M^{A}-\sqrt{\lambda}(1-\rho) T^{A}\right) \\
& -\frac{\left\langle\phi^{2}\right\rangle-\langle\phi\rangle^{2}+\lambda \rho(1-\rho)}{2} \sum_{\mu A}\left(y_{\mu}^{A}\right)^{2}- \\
& \left.\left.\sum_{A<B} \sum_{\mu} y_{\mu}^{A} y_{\mu}^{B}\left[q^{A B}\left(\left\langle\phi^{2}\right\rangle-\langle\phi\rangle^{2}\right)+r^{A B} \lambda \rho(1-\rho)\right]\right\}\right\rangle_{\sigma}
\end{aligned}
$$




$$
\begin{gathered}
=\ln \left\langle\int _ { \kappa } ^ { \infty } \prod _ { A } d x ^ { A } \int _ { - \infty } ^ { \infty } \prod _ { A } d y ^ { A } \operatorname { e x p } \left\{ i \sum_{A} y^{A}+\right.\right. \\
\quad i \sum_{A} y^{A}(2 \sigma-1)\left(\theta-\langle\phi\rangle M^{A}-\sqrt{\lambda}(1-\rho) T^{A}\right) \\
-\frac{\left\langle\phi^{2}\right\rangle-\langle\phi\rangle^{2}+\lambda \rho(1-\rho)}{2} \sum_{A}\left(y^{A}\right)^{2}- \\
\left.\left.\sum_{A<B} y^{A} y^{B}\left[q^{A B}\left(\left\langle\phi^{2}\right\rangle-\langle\phi\rangle^{2}\right)+r^{A B} \lambda \rho(1-\rho)\right]\right\}\right\rangle_{\sigma},
\end{gathered}
$$

and

$$
\begin{aligned}
G_{2}(x, y, s) \equiv & \frac{1}{N} \ln \left[\int _ { - \infty } ^ { \infty } \prod _ { j A } d W _ { i j } ^ { a A } \operatorname { e x p } \left\{-\frac{i}{2} \sum_{A} y^{A} \sum_{j}\left(W_{i j}^{a A}\right)^{2}-i \sum_{A<B} x^{A B} \sum_{j} W_{i j}^{a A} W_{i j}^{a B}\right.\right. \\
& \left.\left.\quad-i \sum_{A} s^{A} \sum_{j} W_{i j}^{a A}\right\}\right] \\
= & \ln \left[\int_{-\infty}^{\infty} \prod_{A} d W^{A} \exp \left\{-\frac{i}{2} \sum_{A} y^{A}\left(W^{A}\right)^{2}-i \sum_{A<B} x^{A B} W^{A} W^{B}-i \sum_{A} s^{A} W^{A}\right\}\right] .
\end{aligned}
$$

The so-called replica symmetric solution is found by taking $q^{A B} \equiv q$, $r^{A B} \equiv r, F^{A B} \equiv F$ and $H^{A B} \equiv H$ for all $A<B$, and setting $z^{A} \equiv z$, $U^{A} \equiv U, E^{A} \equiv E, C^{A} \equiv C, M^{A} \equiv M$ and $T^{A} \equiv T$, for all $A$. In terms of replica symmetric variables, $G_{2}$ has the form:

$$
G_{2}(x, y, s) \approx-\frac{n}{2} \ln (i y-i x)-\frac{1}{2} \frac{n x}{y-x}-\frac{n s^{2}}{i y-i x}+O\left(n^{2}\right)
$$

while $G_{1}$ can be reduced to:

$$
G_{1} \approx n \int_{-\infty}^{\infty} \mathcal{D} s\left\{\rho \ln I_{-}+(1-\rho) \ln I_{+}\right\}+O\left(n^{2}\right)
$$

where,

$$
I_{ \pm}=\frac{1}{2} \operatorname{erfc}\left(\frac{\kappa \pm v+\sqrt{q\left(\left\langle\phi^{2}\right\rangle-\langle\phi\rangle^{2}\right)+r \lambda \rho(1-\rho)} s}{\sqrt{2\left[(1-q)\left(\left\langle\phi^{2}\right\rangle-\langle\phi\rangle^{2}\right)+(1-r) \lambda \rho(1-\rho)\right]}}\right)
$$


and we have set $\mathcal{D} s \equiv e^{-s^{2} / 2} / \sqrt{2 \pi}, v \equiv \theta-\langle\phi\rangle M-\sqrt{\lambda}(1-\rho) T$. $\operatorname{erfc}(\mathrm{z})$ is the complimentary error function: $\operatorname{erfc}(z) \equiv(2 / \sqrt{\pi}) \int_{z}^{\infty} d y e^{-y^{2}}$. Since the integrand of Eq. 23 grows exponentially with $N$, we can evaluate the integral using steepest descent techniques. The saddle point equations which need to be satisfied are:

$$
\begin{gathered}
\frac{\partial G}{\partial E}=0, \quad \frac{\partial G}{\partial C}=0, \quad \frac{\partial G}{\partial z}=0, \quad \frac{\partial G}{\partial U}=0, \quad \frac{\partial G}{\partial F}=0, \quad \frac{\partial G}{\partial H}=0, \\
\frac{\partial G}{\partial q}=0 \quad \text { and } \quad \frac{\partial G}{\partial r}=0 .
\end{gathered}
$$

Solving this set of equations yields a system of three equations which define $q, r$ and $v$ in terms of $\alpha$ and $\lambda$. A little reflection reveals that when $\alpha=P / N$ approaches its critical value, $\alpha_{c}=P_{c} / N$, then $q \rightarrow 1$ and $r \rightarrow 1$, hence, this limit will yields the critical information capacity. From Eq. 32 the following relationship between $q$ and $r$ as they both approach 1 can be deduced:

$$
1-r \approx(1-q) \sqrt{\frac{\left\langle\phi^{2}\right\rangle-\langle\phi\rangle^{2}}{\rho(1-\rho)}} .
$$

We can now write the information capacity per weight as:

$$
\begin{aligned}
\mathcal{E} & =\left[-\rho \ln _{2} \rho-(1-\rho) \ln _{2}(1-\rho)\right] \frac{Q P N}{Q N^{2}+N Q^{2}} \\
& =\left[-\rho \ln _{2} \rho-(1-\rho) \ln _{2}(1-\rho)\right] \frac{\alpha_{c}}{1+\lambda},
\end{aligned}
$$

with:

$$
\begin{aligned}
\alpha_{c}^{-1}=\left(\rho\left\{(K-V) \frac{e^{-(K-V)^{2} / 2}}{\sqrt{2 \pi}}+\frac{1}{2}\left[1+(K-V)^{2}\right] \operatorname{erfc}\left(\frac{-K+V}{\sqrt{2}}\right)\right\}+\right. \\
\left.(1-\rho)\left\{(K+V) \frac{e^{-(K+V)^{2} / 2}}{\sqrt{2 \pi}}+\frac{1}{2}\left[1+(K+V)^{2}\right] \operatorname{erfc}\left(\frac{-K-V}{\sqrt{2}}\right)\right\}\right) \times \\
\\
{\left[1+\lambda \frac{\rho(1-\rho)}{\left\langle\phi^{2}\right\rangle-\langle\phi\rangle^{2}}\right] /\left[1+\lambda \sqrt{\frac{\rho(1-\rho)}{\left\langle\phi^{2}\right\rangle-\langle\phi\rangle^{2}}}\right]^{2}, }
\end{aligned}
$$

where $\mathrm{V}$ is implicitly defined through: 


$$
\begin{aligned}
& \rho\left\{\frac{e^{-(K-V)^{2} / 2}}{\sqrt{2 \pi}}+\frac{K-V}{2} \operatorname{erfc}\left(\frac{-K+V}{\sqrt{2}}\right)\right\}= \\
& (1-\rho)\left\{\frac{e^{-(K+V)^{2} / 2}}{\sqrt{2 \pi}}+\frac{K+V}{2} \operatorname{erfc}\left(\frac{-K-V}{\sqrt{2}}\right)\right\},
\end{aligned}
$$

and $K \equiv \kappa /\left[\left\langle\phi^{2}\right\rangle-\langle\phi\rangle^{2}+\rho(1-\rho)\right]$. Note: For a given $\rho$, the maximum value of $\mathcal{E}$ occurs when $K=0$. By setting $K$ and $\lambda$ equal to zero, one recovers Eq. 4 and 5 in the text. (Its also interesting to note, that since $V=[\theta-\langle\phi\rangle M-\sqrt{\lambda}(1-\rho) T] /\left[\left\langle\phi^{2}\right\rangle-\langle\phi\rangle^{2}+\rho(1-\rho)\right], M$ and $T$, which represent the average values of the inter and intra neuron weights respectively, are not uniquely determined, rather solving Eq. 36 for $V$ only fixes the difference between $T$ and $M$. Furthermore, the threshold $\theta$ can be easily absorbed into either $M$ or $T$ provided either $\langle\phi\rangle \neq 0$ or $\rho \neq 1$.)

We arrived at equations 35 and 36 using the saddle point conditions of Eq. 31 and 32. As the reader can readily verify, these saddle point equations are also locally stable. Furthermore, since the volume of the space of allowable weights is connected and tends to zero as $q, r \rightarrow 1$, the locally stable solution we have found must be the unique solution [4], Therefore, in this case, the replica symmetric solution is also the exact solution. 